\title{
Trabeculectomy outcomes in advanced glaucoma in Nigeria
}

N. Anand

C. Mielke

Department of

Ophthalmology

Luton \& Dunstable Hospital

Luton, UK

V.K. Dawda

Paybody Eye Unit

Coventry \& Warwickshire

Hospital

Coventry, UK

Mr Nitin Anand, MD

(Ophth), FRCS

Department of

Ophthalmology

Luton \& Dunstable Hospital

Lewsey Road

Luton LU4 ODZ, UK

e-mail:

n-anand@ntlworld.com

This paper was presented in part as a poster at the Royal College of Ophthalmologists Annual Congress,

Harrogate, 2000

Received: 9 June 2000 Accepted in revised form: 10 November 2000

\begin{abstract}
Background Trabeculectomy remains the mainstay of therapy for advanced glaucoma in Nigeria due to the unavailability and expense of topical therapy. Little is known of the medium- to long-term outcomes of trabeculectomy in West Africa.

Purpose To retrospectively assess outcomes, in terms of lowering of the intraocular pressure and preserving the visual acuity, and the safety of trabeculectomy in patients with advanced glaucoma in Nigeria.

Methods A retrospective case-note search was carried out from operating theatre records in a private hospital at Lagos, Nigeria from 1989 to 1997. Patients undergoing primary

trabeculectomy with a minimum follow-up of 6 months were included in the study. Visiting consultants and registrars from the UK performed the surgery. Descriptive statistics and life-table analysis were applied to the data. Results One hundred and forty-two eyes of $\mathbf{1 0 0}$ patients were included in the study. When the

(IOP) of less than $22 \mathrm{mmHg}, 30 \%$ reduction from pre-operative levels and a decrease in visual acuity of less than 3 Snellen chart lines, then by life-table analysis success rates were $85 \%, 82 \%$ and $71 \%$ at the 1,2 and 5 year postoperative intervals respectively. Success rates were lower if an IOP of less than $16 \mathrm{mmHg}$ was taken as one of the criteria $(65 \%, 61 \%$ and $46 \%$ at the 1, 2 and 5 year intervals, respectively). Conclusions Trabeculectomy without antimetabolite use appears to be an effective way to lower the IOP of advanced glaucoma patients in Nigeria to less than $22 \mathrm{mmHg}$ but not to less than $16 \mathrm{mmHg}$. The procedure, in experienced hands, is relatively safe with few major complications.
\end{abstract}

4-8 times more likely to have glaucoma than whites. $^{1-3}$ They have an earlier age of onset of the disease, ${ }^{4}$ are more likely to have field loss a a given intraocular pressure (IOP) ${ }^{4,5}$ and more likely to be blind due to glaucoma compared with the white population. ${ }^{1-3}$

Late presentation is common in West Africa Lack of appropriate screening facilities and ignorance about the disease are contributory. Patients usually present when central vision is affected in one or both eyes. Treatment is difficult due to the unavailability and expense of glaucoma medication. Limited compliance with medical therapy and poor follow-up rates seriously hamper treatment. ${ }^{6}$ This makes surgery for glaucoma an attractive option.

Data on trabeculectomy outcomes from this region are scanty, with poor statistical analysis and very limited follow-up. ${ }^{6-10}$ The emphasis has been on intraocular pressure (IOP) rather than visual fields or visual acuity.

The aim of this study was to retrospectively assess outcomes, in terms of lowering the IOP and preserving visual acuity, and the safety of trabeculectomy in eyes with advanced glaucoma in Nigeria.

Key words Africa, Cataract, Glaucoma, Surgery, Trabeculectomy

Open angle glaucoma is a major cause of blindness in West Africa. Data available from the United States and Barbados would suggest that blacks (mostly of West African origin) are

\section{Methods}

All surgery and follow-up examinations were done at the Maja Eye Hospital in Lagos, Nigeria A retrospective case-note search was done from the operating room records between 1988 and 1997. Surgeons based in the UK (consultants and registrars) performed the trabeculectomies. Local doctors saw patients on post-operative visits. Trabeculectomy with intraoperative use of antimetabolites was excluded. Only patients with primary open angle glaucoma undergoing primary trabeculectomy were considered eligible for the study. Patients with a successful outcome but with a follow-up period of less than 6 months were also excluded.

Data were collected on standardised forms and included patient details, ocular diagnosis, coexisting ocular pathology, previous ocular surgery, severity of glaucoma damage as adjudged by disc cupping and visual field loss, pre-operative IOP, visual acuity and 
medications. Operative details and intraoperative complications were noted. The following were recorded at each post-operative visit: visual acuity, IOP, complications and medications. Subsequent surgery for glaucoma and cataract was also noted. Criteria for success were an IOP reduction of more than $30 \%$ from pre-operative levels, a permanent decrease in visual acuity of 2 Snellen chart lines or less from pre-operative levels and IOP of less than either $22 \mathrm{mmHg}$ or $16 \mathrm{mmHg}$ (criterion 2) with or without medication. If visual field progression was clearly evident then the surgery was considered a failure irrespective of IOP level and visual acuity.

Success rates were summarised by the actuarial lifetable analysis method. Comparison of the time to failure of trabeculectomy by the two criteria and the risk factor of age less than 50 years was done in a univariate fashion, by Kaplan-Meier survival curve analyses and the Mantel-Haenszel log-rank test. In patients who had bilateral trabeculectomies, only the eye operated on first was considered for the survival analysis.

\section{Surgical technique}

All patients underwent a Cairns-type trabeculectomy ${ }^{11}$ with slight modifications in technique. A fornix- or limbal-based conjunctival flap was dissected followed by dissection of a triangular or rectangular scleral flap. The exact dimensions of the scleral flap varied according to surgeon. After the excision of a block of corneo-scleral tissue a peripheral iridectomy was done and the scleral flap was closed with one to five 10.0 nylon sutures. The conjunctival flap was closed with 10.0 nylon or 8.0 polyglactin sutures. Subconjunctival bethamethasone 1\% and an antibiotic were given post-operatively. Patients used a topical steroid-antibiotic for at least 4 weeks postoperatively.

\section{Results}

One hundred and thirty-four patients and 189 eyes were identified from the operating theatre records. One hundred patients and 142 eyes were included in the study. Thirty-two eyes of 24 patients who had uncomplicated trabeculectomy were excluded as followup was less than 6 months. Thirteen eyes of 8 patients were excluded as they had had previous intraocular surgery. Also excluded were 1 eye each of patients with congenital glaucoma and acute angle closure glaucoma. Average follow-up was 3 years. Patient details are shown in Table 1. Age less than 50 years has been considered to

Table 1. Demographics

\begin{tabular}{lc}
\hline No. of patients & 100 \\
No. of eyes & 142 \\
Male/female & $71 / 29$ \\
Age (years) & $62(64)^{\mathrm{a}}$ \\
Left/right eye & $81 / 61$ \\
Follow-up (months) & $33.7(26.5)^{\mathrm{a}}$ \\
\hline
\end{tabular}

\footnotetext{
${ }^{\mathrm{a}}$ Mean (median).
}

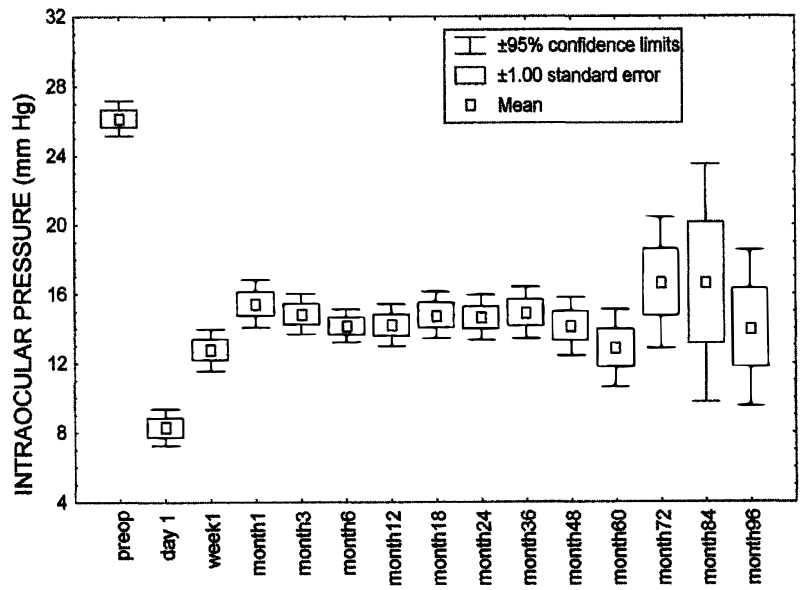

Fig. 1. IOP changes after trabeculectomy.

be a risk factor. ${ }^{12}$ Nineteen eyes $(13 \%)$ of 12 patients (12\%) were in this category (age less than 50 years). Eighty-four per cent of eyes had advanced glaucoma defined as visual field loss either encroaching or involving central vision.

IOP changes are shown in Fig. 1. Average preoperative intraoperative pressure was $26.08 \mathrm{mmHg}$ (range $28-44 \mathrm{mmHg}$ ). Life-table analysis showed that cumulative success rates by the first criterion (IOP less than $22 \mathrm{mmHg}$ and visual acuity loss of 2 lines or less) were $85 \%$ at the end of 1 year, falling to $71 \%$ in 5 years. By the second criterion (IOP less than $16 \mathrm{mmHg}$ and visual acuity loss of 2 lines or less) success rates were much lower, being $65 \%$ at 1 year and $46 \%$ at 5 years. Failure of surgery as defined occurred most frequently in the first 6 months after surgery but continued at a steady rate throughout the follow-up period.

Univariate analysis of survival curves (log-rank test) showed that age less than 50 years did not appear to affect the trabeculectomy outcomes when the success criteria included an IOP less than $22 \mathrm{mmHg}(p=0.45)$. When the success criteria included an IOP less than $16 \mathrm{mmHg}$ then patients with age less than 50 years did significantly worse $(p=0.009)$.

Visual acuity decreased during the follow-up period. Average loss ( \pm standard error) of Snellen chart lines was $0.62 \pm 0.15$ by the end of follow-up. Pre-operatively $55.4 \%$ (79 eyes) of the eyes had a visual acuity of $6 / 12$ or

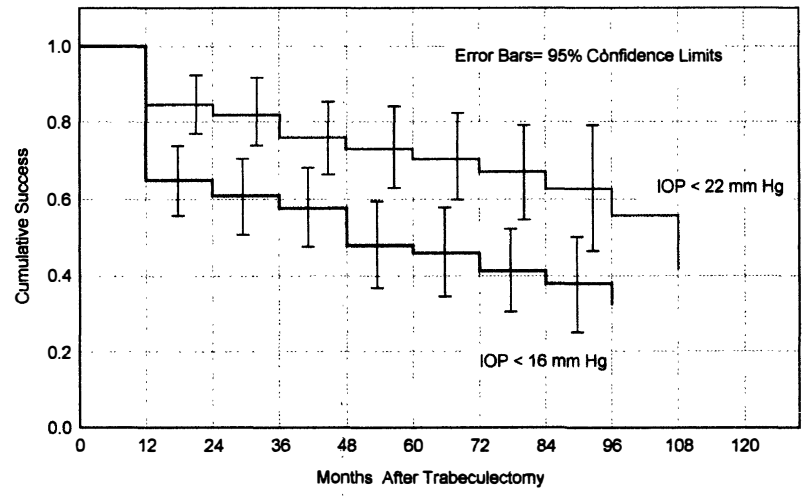

Fig. 2. Comparative cumulative success of trabeculectomy: IOP less than $22 \mathrm{mmHg}$ versus IOP less than $16 \mathrm{mmHg}$. 
Table 2. Visual acuity loss of more than 2 Snellen chart lines after trabeculectomy

\begin{tabular}{lc}
\hline & No. of eyes $(\%)$ \\
\hline Progression of field loss ${ }^{\mathrm{a}}$ & $10(7.0)$ \\
Progression of cataract & $4(2.8)$ \\
Macular degeneration & $1(0.7)$ \\
Diabetic maculopathy & $1(0.7)$ \\
Malignant glaucoma & $1(0.7)$ \\
Total & $17(12.0)$ \\
\hline
\end{tabular}

${ }^{\mathrm{a}} \mathrm{Six}$ of the 10 eyes had an IOP less than $16 \mathrm{mmHg}$.

better and at the end of the follow-up and 31.7\% (43 eyes) retained $6 / 12$ or better. The number of eyes with vision of $6 / 60$ or less increased from $30 \%$ (42 eyes) preoperatively to $38.6 \%$ (54 eyes) at the end of follow-up.

Visual acuity loss of more than 2 Snellen lines was seen in 17 eyes $(12 \%)$ of 16 patients as illustrated in Table 2. Progression of visual field loss to involve central vision was the commonest cause seen in 10 eyes (7\%). Six of these 10 eyes had an IOP less than $16 \mathrm{mmHg}$. Progression of cataract was deemed to be the cause in 4 eyes $(2.8 \%)$. Patients had refused cataract surgery in these cases.

Complications are shown in Table 3. Reformation of a flat anterior chamber was necessary in only 1 case. This patient had presented 1 month after the trabeculectomy with a history of trauma to the eye. A flat chamber and extrachoroidal haemorrhage was observed and he subsequently underwent a scleral tap, vitrectomy, cataract extraction and an anterior chamber intraocular lens implant. Wound leaks were transient and resolved spontaneously. Re-suturing of the conjunctiva was required in only 1 case a week after the trabeculectomy. Malignant glaucoma was recognised in one case during the operative procedure by a persistently flat anterior chamber and the patient subsequently underwent extracapsular cataract extraction with a posterior chamber intraocular lens implant and vitreous aspiration. Iris incarceration in the sclerostomy was seen in 4 eyes (2.8\%). Two cases required further surgery (iridectomy) to unblock the sclerostomy. The one case of blebitis responded well to topical and systemic antibiotics and IOP control was maintained in this case. No endophthalmitis was observed in this cohort. Life-

Table 3. Complications of trabeculectomy No. of eyes (\%)

Minor

Shallow anterior chamber

Hyphaema

18 (12.7)

Transient choroidal detachments

4 (2.8)

Transient wound leaks

4 (2.8)

Major

Damage to lens during surgery

2 (1.4)

Vitreous loss during surgery $^{\mathrm{a}}$

$1(0.7)$

Malignant glaucoma

2 (1.4)

Blebitis

$1(0.7)$

Iris incarceration in sclerostomy

4 (2.8)

Extrachoroidal haemorrhage

Endophthalmitis

$1(1.4)$

${ }^{\mathrm{a}}$ Aphakic patient.

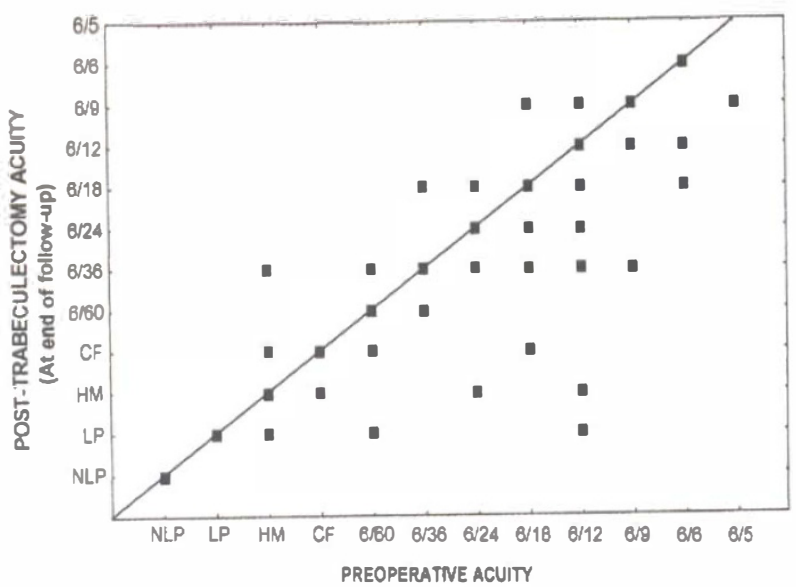

Fig. 3. Visual acuity changes after trabeculectomy (Snellen chart).

table analysis showed that at 5 years, the cumulative probability of undergoing cataract surgery was $29 \%$. Twenty-eight eyes (19.7\%) had a cataract extraction during the follow-up period.

Glaucoma surgery was repeated in 16 eyes (11.3\%). Combined cataract and trabeculectomy was done in 3 eyes, trabeculectomy in 6 eyes and trabeculectomy augmented with 5 -fluorouracil (5FU) in 4 eyes. Two eyes underwent two repeat trabeculectomies augmented with intraoperative $5 \mathrm{FU}$.

\section{Discussion}

This study contributes valuable information regarding outcomes of primary trabeculectomy without antimetabolite use in West Africa. Although it is retrospective and subject to the well-known associated problems of reliable data collection we believe our efforts to record meaningful pre- and post-operative data have been successful. There is inevitably some heterogeneity in techniques as the study involved trabeculectomy done by a number of general ophthalmic surgeons. With the exception of eyeball massage, no post-operative manipulation of the filtering bleb was done. We feel that this may have had an impact on the outcomes. Most failures occurred within the first 6 months. Experienced glaucoma surgeons would have initiated early postoperative manipulation of the filtering bleb, probably resulting in higher success rates.

Serious complications were infrequent. Damage to the lens while performing the peripheral iridectomy in 1 case was attributed to the relative inexperience of the surgeon, a registrar. Endophthalmitis was not observed in this cohort.

At the end of follow-up, the mean visual acuity of the entire group was reduced by approximately 1 Snellen chart line compared with pre-operative values (Fig. 3). This is in agreement with other reports, which show that reduction of visual acuity is a common event in the long term after trabeculectomy. ${ }^{13-15}$ It has been argued that even without an operation, glaucoma patients would experience a deterioration of best-corrected visual acuity. ${ }^{16,17}$ In patients operated on for glaucoma the two common causes for decrease in vision are development 
Table 4. Trabeculectomy outcomes in West Africa: previous reports ${ }^{6-10}$

\begin{tabular}{|c|c|c|c|c|c|c|c|}
\hline Reference & Year & Place & $\begin{array}{c}\text { No. of } \\
\text { patients }\end{array}$ & Follow-up & Success criteria & $\begin{array}{l}\text { Successful } \\
\text { outcomes }\end{array}$ & $\begin{array}{l}\% \text { lost to } \\
\text { follow-up }\end{array}$ \\
\hline Chatterjee and Ansari ${ }^{7}$ & 1972 & Ghana & 24 & $1-12$ months & IOP $<21 \mathrm{mmHg}$ & $79 \%$ & $19 \%$ \\
\hline Kietzman $^{8}$ & 1976 & Nigeria & 221 & $>4$ months & IOP $<21 \mathrm{mmHg}$ & $74 \%$ & $60 \%$ \\
\hline Sanford-Smith ${ }^{9}$ & 1978 & Nigeria & 51 & $?$ & IOP $<21 \mathrm{mmHg}$ & $65 \%$ & $37 \%$ \\
\hline Thommy and Bhar ${ }^{10}$ & 1979 & Nigeria & 111 & 6-19 months & IOP $<20 \mathrm{mmHg}$ & $95 \%$ & $20 \%$ \\
\hline Verrey et al. ${ }^{6}$ & 1990 & Ghana & 188 & 6 months & IOP $<22 \mathrm{mmHg}$ & $84 \%$ & $83 \%$ \\
\hline \multirow[t]{2}{*}{ Present study } & 2000 & Nigeria & 100 & Av. 33 months & 1. IOP $<22 \mathrm{mmHg}$ & $65 \%$ at 5 years & $17 \%$ less than \\
\hline & & & & & 2. IOP $<16 \mathrm{mmHg}$ & $46 \%$ at 5 years & 6 months $^{\mathrm{a}}$ \\
\hline
\end{tabular}

${ }^{\mathrm{a}}$ Excluded from analyses.

or progression of cataract or progressive central field loss. We paid particular attention to visual acuity changes as most patients (85\%) had advanced glaucomatous field loss, which encroached on fixation. Lack of trained personnel to conduct the field tests and poor patient comprehension limited the value of visual field tests in this study. Therefore it was difficult to interpret the field tests for most patients except for those in whom field loss progressed to involve central vision. In any case diagnosis of visual field progression remains difficult, particularly in eyes with advanced field loss, due to long-term fluctuation of fields. ${ }^{18}$

Cataracts depress visual acuity more than visual field. ${ }^{14}$ Due to the retrospective nature of this study it is not possible to determine accurately the effect trabeculectomy had on the development of cataract in operated eyes. The cumulative probability of having cataract extraction was $29 \%$ at 5 years in this study, broadly in concurrence with previous reports from other parts of the world. ${ }^{13,14,19-21}$ It is important to note that this may be an underestimate as 4 patients refused surgery and it is possible others may have had surgery elsewhere.

Higher follow-up rates were observed in this study compared with others ${ }^{6-10}$ from this region, probably due to the higher literacy and affluence of the patients. Also this study was conducted in the largest city in Nigeria where means of transportation are relatively better.

Comparisons between this study and studies on trabeculectomy outcomes in blacks in other parts of the world will be misleading for various reasons including differing criteria for success, technical skills of operating personnel, post-operative management and follow-up duration. One of the biggest problems in most studies is the lack of adequate follow-up ${ }^{6-10}$ (Table 4). If most patients are lost to follow-up immediately after surgery, it is impossible to know whether the majority of the defaulters are successes or failures, resulting in a biased study. We were unable to find in the literature a study originating from West Africa, or for that matter anywhere in Africa, which took this factor into account. Survival curve or life-table analysis is a useful statistical tool to study outcomes in operated eyes when not all the patients have been followed for the entire study period. ${ }^{22}$ In this study, by considering the censored data (patients for whom no complete data were available because they were lost to follow-up or were still being followed), we could more accurately estimate survival times at different points over time. Black race is considered a risk factor for failure of surgery, ${ }^{23,24}$ though there is no definite evidence for this. Broadway et al. ${ }^{25}$ reported that conjunctiva from black patients in the UK contained a greater number of macrophages and possibly fibroblasts in comparison with white patients. They also found lower success rates in blacks but this difference was not significant.

Most previous reports have used $21 \mathrm{mmHg}$ or less as a criterion for 'success' in reporting glaucoma surgery outcomes. Available evidence suggests, however, that if there is a safe upper limit for IOP in 'high tension' glaucoma patients it is probably less than $16 \mathrm{mmHg}^{26}$ However, as was the case in 6 of the 10 eyes in this study with progressive field loss, in advanced glaucoma visual field loss progression continues at IOPs lower than $16 \mathrm{mmHg}$.

The use of antimetabolites has greatly enhanced the success rates of drainage surgery in all patients irrespective of risk factors for failure. Mermoud et al..$^{27}$ in a prospective study on South African black patients showed significantly higher success rates with intraoperative use of mitomycin $C$ compared with a control group. They also found a higher incidence of cystic avascular blebs and late bleb leaks in the mitomycin group. The risk of endophthalmitis after trabeculectomy is probably higher with antimetabolite use. Endophthalmitis is also associated with cystic, thinwalled blebs as seen after mitomycin C use. ${ }^{28-30}$ In another prospective study, though with a limited followup, Egbert et al..$^{31}$ reported that a single intraoperative application of 5FU markedly improved the IOP lowering effect of trabeculectomy in a West African population. The same group (Singh et al. ${ }^{32}$ ) demonstrated that intraoperative mitomycin $\mathrm{C}$ application was as safe as but more efficacious than intraoperative application of 5 FU in lowering IOP in the short term. However, they also found that mitomycin $\mathrm{C}$ use did not result in a significantly higher proportion of eyes achieving an IOP of less than $15 \mathrm{mmHg}$. Remarkably, they also reported that hypotony (IOP less than $5 \mathrm{mmHg}$ ) was seen in only 2 of 10 eyes following trabeculectomy with 5FU or mitomycin C. No case of hypotony-related maculopathy was seen and they concluded that this disorder is rare in black West Africans compared with whites. ${ }^{33}$

In conclusion, trabeculectomy without antimetabolite use appears to be an effective way to lower the IOP of advanced glaucoma patients in Nigeria to less than 
$22 \mathrm{mmHg}$ but not to less than $16 \mathrm{mmHg}$. The procedure in experienced hands is relatively safe with few major complications. Cataract progression resulting in further diminution of visual acuity remains a concern and at least $29 \%$ of these patients will require cataract extraction within 5 years.

\section{References}

1. Sommer A, Tielsch JM, Katz J, et al. Relationship between intraocular pressure and primary open angle glaucoma among white and black Americans. Arch Ophthalmol 1991;109:1090-5.

2. Sommer A, Tielsch JM, Katz J, et al. Racial differences in the cause-specific prevalence of blindness in east Baltimore. $\mathrm{N}$ Engl J Med 1991;325:1412-7.

3. Leske MC, Connell AM, Schachat AP, et al. The Barbados Eye Study: prevalence of open angle glaucoma. Arch Ophthalmol 1994;112:821-9.

4. The Advanced Glaucoma Intervention Study (AGIS). 3. Baseline characteristics of black and white patients. Ophthalmology 1998;105:1137-45.

5. The Glaucoma Laser Trial (GLT). 5. Subgroup differences at enrolment. Glaucoma Laser Trial Research Group. Ophthalmic Surg 1993;24:232-40.

6. Verry JD, Foster A, Wormald R, et al. Chronic glaucoma in Northern Ghana: a retrospective study of 397 patients. Eye 1990;4:115-20.

7. Chatterjee S, Ansari MW. Microsurgical trabeculectomy in Ghana. Br J Ophthalmol 1972;56:783-7.

8. Kietzman B. Glaucoma surgery in Nigerian eyes: a five-year study. Ophthalmic Surg 1976;7:52-8.

9. Sandford-Smith JH. The surgical treatment of open-angle glaucoma in Nigerians. Br J Ophthalmol 1978;62:283-6.

10. Thommy CP, Bhar IS. Trabeculectomy in Nigerian patients with open-angle glaucoma. Br J Ophthalmol 1979;63:636-42.

11. Cairns JE. Trabeculectomy: preliminary report of a new technique. Am J Ophthalmol 1968;66:673-9.

12. Sturmer J, Broadway D, Hitchings R. Young patient trabeculectomy: assessment of risk factors. Ophthalmology 1993;100:928-39.

13. Nouri-Mahdevi K, Brigatti M, Weitzman M, et al. Outcomes of trabeculectomy for primary open angle glaucoma. Ophthalmology 1995;102:1760-9.

14. The Advanced Glaucoma Intervention Study (AGIS). 4. Comparison of treatment outcomes within race: seven-year results. Ophthalmology 1998;105:1146-63.

15. Mills KB. Trabeculectomy: long-term follow-up. Br J Ophthalmol 1981;65:636-42.

16. Jay JL, Allan D. The benefit of early trabeculectomy versus conventional management in primary open angle glaucoma. Eye 1989;3:528-35.
17. Midgal C, Gregory W, Hitchings R. Long-term functional outcome after early surgery compared with laser and medicine in open angle glaucoma. Ophthalmology 1994;101:1651-7.

18. Boeglin RJ, Caprioli J, Zuluaf M. Long-term fluctuation of visual field in glaucoma. Am J Ophthalmol 1992;113:396-400.

19. Jerndal $T$, Lundstrom $M$. Three hundred and thirty trabeculectomies: a long time study ( $3-5 \frac{1}{2}$ years). Acta Ophthalmol (Copenh) 1980;58:947-56.

20. Tornqvist G, Drolsum LK. Trabeculectomies: a long-term study. Acta Ophthalmol (Copenh) 1991;69:450-4.

21. Chen TC, Wilensky JT, Viana MA. Long-term follow-up of initially successful trabeculectomy. Ophthalmology 1997;104:1120-5.

22. Glantz SA. How to analyse survival data. In: Primer of biostatistics. 4th edn. New York: McGraw-Hill, 1997:373-402.

23. Shingleton BJ, Distler JA, Baker BH. Filtration surgery in black patients: early results in a West Indian population. Ophthalmic Surg 1987;18:195-9.

24. Wilson MR. Posterior lip sclerectomy vs trabeculectomy in West Indian blacks. Arch Ophthalmol 1989;107:1604-8.

25. Broadway D, Grierson I, Hitchings R. Racial differences in the results of glaucoma filtration surgery: are racial differences in the conjunctival cell profile important? $\mathrm{Br} \mathrm{J}$ Ophthalmol 1994;78:466-75.

26. Mao LK, Stewart WC, Shields MB. Correlation between intraocular pressure control and progressive glaucomatous damage in primary open-angle glaucoma. Am J Ophthalmol 1991;111:51-5.

27. Mermoud A, Salmon JF, Murray AND. Trabeculectomy with mitomycin $\mathrm{C}$ for refractory glaucoma in Blacks. Am J Ophthalmol 1993;116:72-8.

28. Wolner B, Liebmann JM, Sassani JW, Ritch R, Speaker M, Marmor M. Late bleb-related endophthalmitis after trabeculectomy with adjunctive 5-fluorouracil. Ophthalmology 1991;98:1053-60.

29. Solomon A, Ticho U, Frucht-Pery J. Late-onset, blebassociated endophthalmitis following glaucoma filtering surgery with or without antifibrotic agents. J Ocul Pharmacol Ther 1999;15:283-93.

30. Mochizuki K, Jikihara S, Ando Y, Hori N, Yamamoto T, Kitazawa $Y$. Incidence of delayed onset infection after trabeculectomy with adjunctive mitomycin $\mathrm{C}$ or 5fluorouracil treatment. Br J Ophthalmol 1997;81:877-83.

31. Egbert PR, William AS, Singh K. A prospective trial of intraoperative fluorouracil during trabeculectomy in a black population. Am J Ophthalmol 1993;116:612-6.

32. Singh K, Egbert PR, Byrd S, et al. Trabeculectomy with intraoperative 5-fluorouracil vs mitomycin C. Am J Ophthalmol 1997;123:48-53.

33. Singh K, Byrd S, Egbert PR, Budenz D. Risk of hypotony after primary trabeculectomy with antifibrotic agents in a black west African population. Glaucoma 1998;7:82-5. 